\title{
Bio-Inspired Learning and Adaptation for Optimization and Control of Complex Systems
}

\author{
Jing Na $\left(\mathbb{D},{ }^{1}\right.$ Zhile Yang $\mathbb{D}^{2},{ }^{2}$ Shyam Kamal $\left(\mathbb{D},{ }^{3}\right.$ Liang $\mathrm{Hu}^{4}{ }^{4}$ \\ Wenbo Wang, ${ }^{5}$ and Yimin Zhou ${ }^{6}{ }^{6}$ \\ ${ }^{1}$ Kunming University of Science and Technology, Kunming, China \\ ${ }^{2}$ Queens University Belfast, UK \\ ${ }^{3}$ Indian Institute of Technology (BHU), Varanasi, India \\ ${ }^{4}$ De Montfort University, UK \\ ${ }^{5}$ Nanyang Technological University, Singapore \\ ${ }^{6}$ Chinese Academy of Science, China \\ Correspondence should be addressed to Jing Na; najing25@163.com
}

Received 10 April 2019; Accepted 10 April 2019; Published 23 April 2019

Copyright (C) 2019 Jing Na et al. This is an open access article distributed under the Creative Commons Attribution License, which permits unrestricted use, distribution, and reproduction in any medium, provided the original work is properly cited.

Learning and adaptation play an important role in solving numerous science and engineering problems, including artificial intelligence, control engineering, and many multidisciplinary topics. In this respect, a series of bioinspired methods, such as reinforcement learning, coevolution learning, and approximate dynamic programming as well as swarm evolutions, provide essential theoretical tools for solving various optimization and control problems. This has stimulated great research interests and developments on learning and adaptation. This special issue aims at providing a specific opportunity to review the state-of-the-art of this recently emerging and cross-disciplinary field of bio-inspired learning and adaptation. In this special issue, we bring together researchers to present the latest progress, novel research methodologies, and a broad spectrum of potential research topics.

This special issue has been very successful. We have attracted more than 100 submissions, and after a thorough peer review process, more than 50 papers were accepted for publication. These papers cover important research topics in learning and adaptation for optimization and control, including differential evolution, neural network, adaptive control, system modeling, and identification, with particular emphasis on applications for robotics, UAV, autonomous vehicles, and so on. We believe that the original papers collected in this special issue highlight the emerging and state-of-the-art research topics related to the learning and adaptation and will introduce readers to the latest advances in this field. However, due to the huge number of papers published in this special issue, it is obviously impossible for us to review all papers in this editorial. Only several highlighted papers will be mentioned here, and we refer to the special issue for more details.

Evolutionary algorithms have been proved as an efficient methodology for solving the optimization problem. Y. $\mathrm{Yu}$ et al. proposed a simple and efficient multipopulation management strategy to dynamically adjust the subpopulation number in different phases throughout the evolution. Through determining the subpopulation number, this method maintains the population diversity and enhances the exploration capability. W. Ying et al. suggested an efficient Conical Area Differential Evolution (CADE) algorithm, which employs biased decomposition and dual populations for constrained optimization by borrowing the idea of cone decomposition for multiobjective optimization. B. Wang et al. addressed a maintenance plan optimization problem for building energy retrofitting with a novel multiscale differential evolution based algorithm. B. Xu et al. presented 
a multistrategy based multiobjective differential evolution for optimal control in chemical processes. X. Peng and Y. Wu investigated a Selective Multiple Population (SMP) based Cooperative Coevolution (CC), a.k.a., CC-SMP, to enhance the cooperation of subproblems by addressing two challenges: finding informative collaborators whose fitness and diversity are qualified and adapting to the dynamic landscape.

Neural Networks (NN) have been widely adopted in learning and adaptation for solving the optimization and control problems during the past decades. In this field, $\mathrm{S}$. $\mathrm{Xu}$ et al. introduced a nonlinear autoregressive neural network to address the problem of power loss prediction for aging characteristics as well as condition monitoring for parallel-connected power modules. K. Liang et al. proposed a convolutional recurrent neural network for fault diagnosis of high-speed train bogie, and C. Lin et al. adopted a recurrent neural network to achieve obstacle avoiding for unmanned underwater vehicles. M. Liang et al. also used a recurrent neural network to solve the optimization problem for prediction of $\mathrm{R} 245 \mathrm{Fa}$ flow boiling heat transfer inside horizontal smooth tubes. X. Gao and R. Liu suggested a multiscale Chebyshev neural network for identification and adaptive control of systems with Backlash-Like hysteresis.

Furthermore, the bio-inspired online learning and adaptation have been incorporated into control designs for various linear and nonlinear systems, which have attracted a large number of researchers in control and automation communities for decades. In this special issue, M. Wan et al. introduced an adaptive sliding mode tracking control mechanism for unmanned autonomous helicopters, where neural networks are used to compensate the uncertainties online. J. Zhang et al. investigated data-driven superheating control of organic Rankine cycle processes, where the Quantized Minimum Error Entropy (QMEE) is adopted to construct the performance index of superheating control systems. C. Zhang et al. suggested a reconfigurable control method based on cascade Active Disturbance Rejection Controller (ADRC) for a hovering Planar Vertical Take-Off and Landing (PVTOL) aircraft. W. Wei also extended the idea of ADRC for disturbance rejection and synchronization of Morris-Lecar neurons and nonlinear systems. C. Ming et al. also addressed the velocity control for air-breathing supersonic vehicles by using an active disturbance rejection scheme. C. Yang et al. proposed a mechanism for modelfree composite control of flexible manipulators by further tailoring the principle of adaptive dynamic programming to achieve optimal control. The optimal control for a nonlinear boiler-turbine unit was considered by G. Zhao et al. in their work, where an adaptive receding Galerkin algorithm was proposed. Meanwhile, Q. Zhu et al. proposed an efficient yet simple U-model based control paradigm for complex dynamic rational systems using a new U-model reformulation.

The practical applications of learning and adaptation for control designs have been a major theme in this special issue. These applications include robotic systems, servo systems, hydraulic actuation, smart grid, multiagent system, and supersonic vehicles, just to mention a few. In this special issue, T. Zeng et al. developed an adaptive finite-time sliding mode control for dual-motor driving systems, where a disturbance observer was adopted to handle uncertainties. S. Li et al. proposed a direct inverse controller for Permanent Magnet Synchronous Motor (PMSM) with a Radial Basis Function NN (RBFNN) as a compensator. The idea of adaptive robust control was further tailored for disturbance compensation and active vibration isolation by B. Zhao et al. in their paper. R. Bai and D. Guo investigated sliding mode control of active suspension systems configured with hydraulic actuator. The hybrid steering control for unmanned driving electric vehicle with in-wheel motors was addressed by $\mathrm{Y}$. Li et al., who proposed to combine Back Propagation NN (BPNN) with Genetic Algorithm (GA) to achieve a better response. $\mathrm{L}$. Xi et al. investigated a novel automatic generation control method for the islanded smart grid by developing a mechanism of ecological population cooperative control. G. Hou et al. studied model predictive control of gas turbine in combined cycle unit, where a fuzzy system is incorporated into the control synthesis. Furthermore, the leader-following consensus control of multiagent systems was studied in the work by X. Hou and Y. Liu, where an event-triggered scheme was suggested and only the output feedback was required.

Finally, the theoretical studies and applications of robotic systems have also attracted significant attentions in this special issue. J. Wu et al. studied the problem on path planning of space robot for on-orbit detection, for which the ideas of GA algorithm, Singular Value Decomposition (SVD), and Damped Least Squares (DLS) were combined to improve the tracking accuracy of a 6-degree-of-freedom free-floating space robot. C. Wang et al. proposed a modeling method for a flexible lower extremity exoskeleton robot and proposed a method of deep locomotion mode identification. C. Wang et al. addressed the problem of terrain adaptive estimation of instantaneous centers of rotation for tracked robot. G. Gao et al. investigated the problem of hybrid optimal kinematic parameter identification and calibration for an industrial robot. In their work, a BPNN was adopted together with Particle Swarm Optimization (PSO) to achieve enhanced performance. N. Wang et al. presented an efficient method for the exploration of muscle fatigue effects by analysing the surface ElectroMyoGraphy (sEMG) signals in the framework of a bio-inspired robot learning scheme.

The selected papers in the special issue could not exhaustively cover all the recent advances of the bio-inspired learning and adaptation methods for optimization and control of complex systems. Nevertheless, they provide an excellent overview of the state-of-the-art progress in this area. We hope these high-quality papers may enrich the knowledge of the complex systems community and provide valuable insight into this topic for the readers.

\section{Conflicts of Interest}

The authors declare that they have no conflicts of interest. 


\section{Acknowledgments}

The guest editors would like to acknowledge and appreciate both the authors and the reviewers for their significant contributions towards the success of this special issue.

Jing $\mathrm{Na}$ Zhile Yang Shyam Kamal Liang $\mathrm{Hu}$ Wenbo Wang Yimin Zhou 


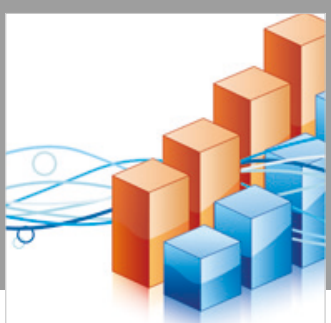

Advances in

Operations Research

\section{-n-m}
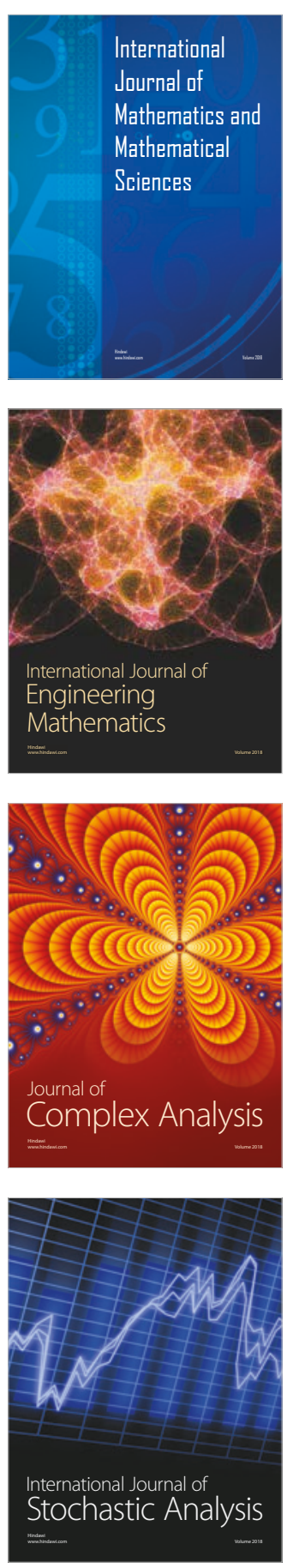
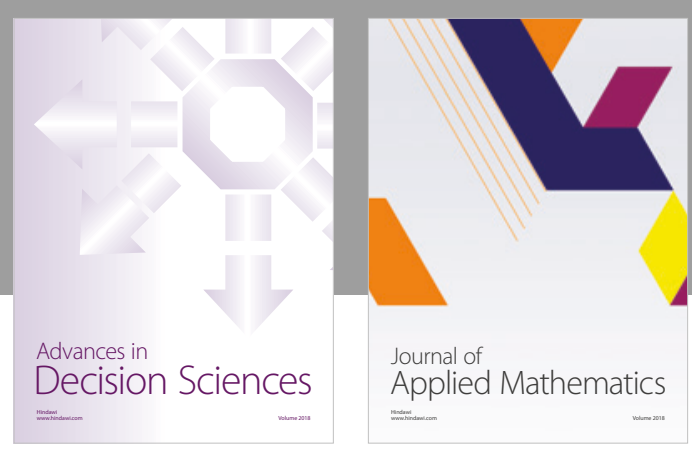

Journal of

Applied Mathematics
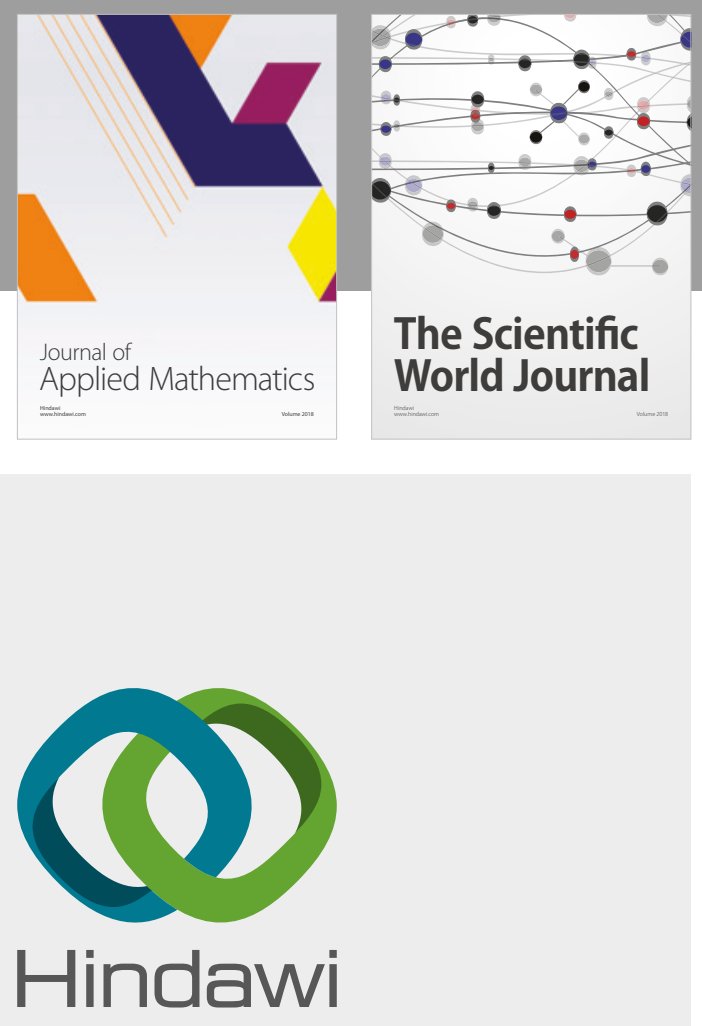

Submit your manuscripts at

www.hindawi.com

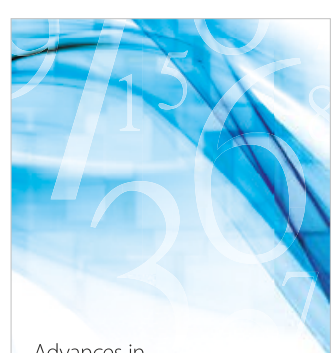

Advances in
Numerical Analysis
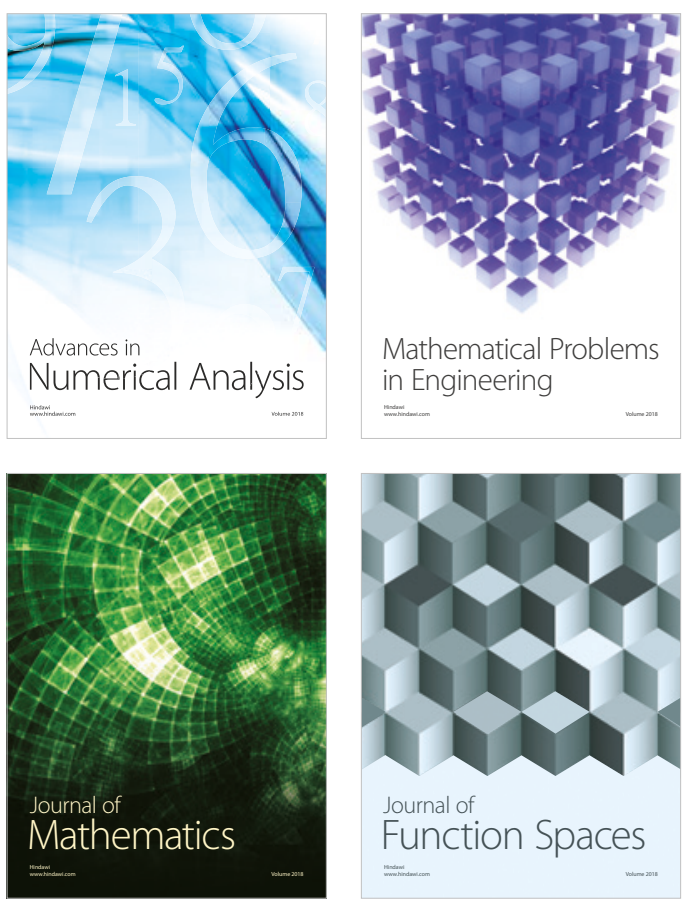

Mathematical Problems in Engineering

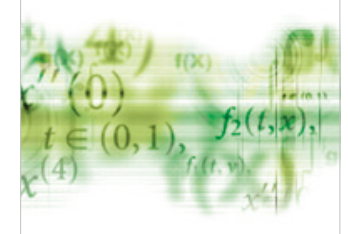

International Journal of

Differential Equations

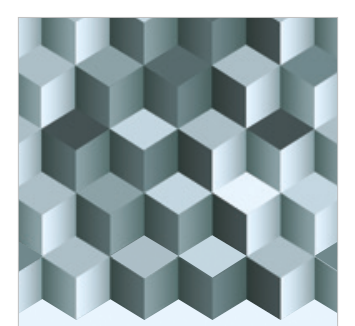

Journal of

Function Spaces
The Scientific

World Journal

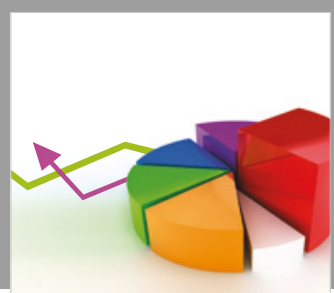

Journal of

Probability and Statistics
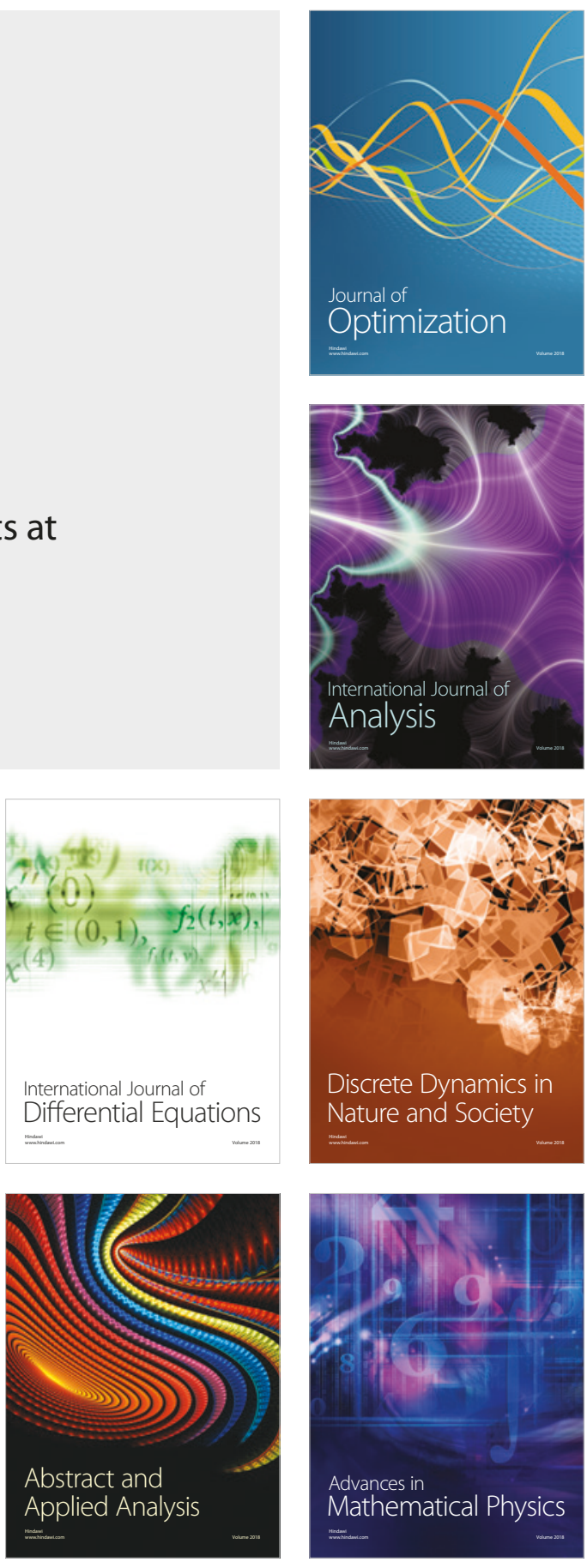